\title{
Parámetros de estabilidad en híbridos de girasol con alto contenido de oléico
}

\section{Stability parameters in sunflower hybrids with high oleic content}

\author{
Tania Lizzeth Guzmán-Silos ${ }^{1}$, Miguel Ángel Gallegos-Robles ${ }^{2 *}$, Juan Ramón Esparza-Rivera ${ }^{3}$, Cirilo \\ Vázquez-Vázquez ${ }^{2}$, Uriel González-Salas ${ }^{2}$, J. Guadalupe Luna-Ortega ${ }^{4}$, Anselmo González-Torres ${ }^{5}$ \\ ${ }^{1}$ Doctorado Institucional en Ciencias Agropecuarias y Forestales. Universidad Juárez del Estado de Durango, Facultad de \\ Agricultura y Zootecnia. \\ ${ }^{2}$ Universidad Juárez del Estado de Durango, Facultad de Agricultura y Zootecnia, Ejido Venecia, CP. 35000 carretera Gómez \\ Palacio-Tlahualilo km 35. \\ ${ }^{3}$ Universidad Juárez del Estado de Durango, Facultad de Ciencias Químicas. Av. Artículo 123 s/n, Fracc. Filadelfia, CP. 35010 \\ Gómez Palacio, Durango. \\ ${ }^{4}$ Universidad Politécnica de la Región Laguna. Ejido Santa Teresa, Municipio de San Pedro de las Colonias, Coahuila. \\ ${ }^{5}$ Universidad Autónoma Agraria Antonio Narro Unidad Laguna. Periférico Raúl López Sánchez s/n Col. Valle Verde CP 27059, \\ Torreón, Coahuila. \\ *Autor de correspondencia: magallegos@ujed.mx
}

Artículo científico recibido: 13 de diciembre de 2015 aceptado: 07 de noviembre de 2016

RESUMEN. Se evaluaron híbridos de girasol que tienen aceite con alto contenido de ácido oléico, los cuales fueron Olisun 1, Olisun 2, Sabritas 1, Híbrido 1, Híbrido 2 e Híbrido 3, en los ambientes de Venecia, Durango, Durango, Durango en el ciclo Primavera-Verano 2010; y Venecia, Durango, en el ciclo Primavera-Verano 2011, bajo un diseño de bloques completos al azar con cuatro repeticiones. El análisis de parámetros de estabilidad, reveló diferencias significativas $(\mathrm{p}<0.05)$ entre híbridos para el contenido de ácido oléico. Para rendimiento de grano el híbrido 1 fue superior $(\mathrm{p}<0.05)$ a los otros híbridos evaluados. El modelo de Eberhart y Russell para las variables ácido oléico y rendimiento clasificó a los híbridos como estables, con excepción del híbrido Sabritas 1 que tuvo desviación de la regresión significativa que lo clasificó como inconsistente. Con base en el contenido de ácido oléico, rendimiento de grano y estabilidad, los mejores híbridos fueron Híbrido 3 y Sabritas 1.

Palabras clave: Ácido oléico, ambientes, estabilidad, Helianthus annuus L., rendimiento

ABSTRACT. Sunflower hybrids that have oil with high oleic acid content (Olisun 1, Olisun 2, Sabritas 1, Hybrid 1, Hybrid 2 and Hybrid 3) were evaluated in the environments of Venecia, Durango and Durango, Durango in the 2010 Spring-Summer cycle, and in Venecia, Durango in the 2011 Spring-Summer cycle, under a randomized complete block design with four replicates. Analysis of stability parameters revealed significant differences $(p<0.05)$ among hybrids for oleic acid content. For grain yield, hybrid 1 was superior $(p<0.05)$ to the other hybrids evaluated. The Eberhart and Russell model for the variables oleic acid and yield classified the hybrids as stable, with the exception of the hybrid Sabritas 1 that had significant deviation of the regression that classified it as inconsistent. Based on oleic acid content, grain yield and stability, the best hybrids were Hybrid 3 and Sabritas 1.

Key words: oleic acid, environments, stability, Helianthus annuus L., yield

\section{INTRODUCCIÓN}

El girasol (Helianthus annuus L.) es un cultivo que tiene como uso principal la extracción de aceite (INIFAP 2015). De los diferentes tipos de aceite que contiene la semilla, sobresale el ácido oléico que tolera la oxidación durante el calentamiento (Ali et al. 2013). Los cultivares tradicionales contienen mayormente ácido linoléico, moderado ácido oléico y bajo ácido linolénico (Sobrino et al. 2003), mientras que los cultivares clasificados como de alto contenido de ácido oléico pueden con- 
tener hasta $89 \%$ de ácido oléico (Alberio et al. 2016). Se sabe que la composición de los ácidos grasos que puedan tener los cultivares depende del genotipo, del manejo agronómico y de las condiciones ambientales (Turhan et al. 2010, Tabrizi 2012).

Dentro de los factores ambientales, los climas cálidos y las temperaturas altas en las noches durante el inicio del llenado de grano, favorecen la formación de altos contenidos de ácido oléico, mientras que climas fríos favorecen altos contenidos de ácido linoléico (Rolletschek et al. 2007, Turhan et al. 2010). Esta respuesta diferencial a las diferentes condiciones ambientales se conoce como interacción genotipo-ambiente, por que un mismo genotipo puede mostrar diferentes comportamientos en diferentes ambientes (Balalic et al. 2011). En la selección y recomendación de híbridos o variedades para su explotación comercial en diferentes ambientes, es necesario considerar que la interacción genotipo-medio ambiente tiene gran importancia, por lo que es necesario la clasificación y caracterización de los genotipos de acuerdo a su comportamiento en diferentes ambientes (Eberhart y Russell 1966). Por lo que la evaluación de cultivares de girasol en diferentes ambientes para probar su adaptación y estabilidad en relación a la consistencia del rendimiento de semilla y composición de ácidos grasos es un paso importante (Van der Merwe et al. 2013). Por lo anterior el objetivo fue identificar la estabilidad de híbridos de girasol con alto contenido de ácido oléico.

\section{MATERIALES Y MÉTODOS}

\section{Material genético}

Se evaluaron los híbridos de girasol Olisun 1, Olisun 2, Híbrido 1, Híbrido 2, Híbrido 3 y Sabritas 1 que tienen alto contenido de ácido oléico. El híbrido Olisun 1 y Olisun 2 tiene contenidos de entre 80 y $89 \%$ de ácido oléico; ambos son híbridos simples, desarrollados en Argentina, que tienen buena tolerancia a plagas y enfermedades, color de grano negro, ciclo intermedio a largo y floración de 75 a 87 d. El híbrido Sabritas 1 y los híbridos 1, 2 y 3 son híbridos simples, adaptados al norte de México, que soportan periodos de sequía, altas temperaturas, buena tolerancia a plagas y enfermedades, con alto potencial de rendimiento, contenidos de ácido oléico entre el 60 y $80 \%$, ciclo intermedio a largo, floración de $55 \mathrm{~d}$ y color de grano negro.

\section{Localidades}

Los materiales se evaluaron en Venecia, Durango, en el ciclo P-V 2010 y ciclo P-V 2011, y en Durango, Durango, en el ciclo P-V 2010. En la localidad de Venecia, Durango, se sembró en la Facultad de Agricultura y Zootecnia de la Universidad Juárez del Estado de Durango, localizada en el km 35 de la Carretera Gómez Palacio - Tlahualilo, temperatura media anual de $22{ }^{\circ} \mathrm{C}$, precipitación de $206 \mathrm{~mm}$, humedad relativa del $50 \%$ y altitud de 1123 msnm. Mientras que en Durango, Durango, se sembró en la Facultad de Medicina y Veterinaria y Zootecnia de la Universidad Juárez del Estado de Durango, localizada en el km 11 de la Carretera Mezquital - Durango, en donde se tiene temperatura media anual de $17{ }^{\circ} \mathrm{C}$, precipitación de $448 \mathrm{~mm}$, humedad relativa de $64 \%$ y altitud de 1 872 msnm (INEGI 2008).

\section{Diseño experimental y manejo agronómico}

Los seis híbridos se evaluaron bajo un diseño experimental de bloques completos al azar con cuatro repeticiones. La unidad experimental constó de cuatro surcos de $4 \mathrm{~m}$ de largo, con distancia entre surcos de $85 \mathrm{~cm}$ y entre plantas de $25 \mathrm{~cm}$, para tener una población aproximada de 47000 plantas $\mathrm{ha}^{-1}$. Las fechas de siembra para los diferentes ambientes fueron junio del 2010 en Durango, julio de 2010 y junio de 2011 en Venecia. Se fertilizó con la dosis 120-80-00 de NPK ha ${ }^{-1}$, aplicando la mitad de $\mathrm{N}$ y el total de $\mathrm{P}$ y $\mathrm{K}$ al momento de la siembra y la otra mitad del $\mathrm{N}$ antes del primer riego de auxilio. Para el control de la palomilla del capítulo (Homoeosoma electellum), se realizó una aplicación preventiva con Ambush 34 CE a razón de $500 \mathrm{ml} \mathrm{ha}^{-1}$. 


\section{Variables evaluadas}

Antes de la floración, se marcaron de forma aleatoria cinco plantas con competencia completa en cada parcela y repetición para la evaluación de las variables. Se evaluó el rendimiento de grano (RG) en $\mathrm{t} \mathrm{ha} \mathrm{a}^{-1}$, como el peso de los aquenios cosechados en la parcela útil, dividido entre el total de plantas cosechadas y multiplicado por el total de plantas por hectárea; altura de planta (AP) en $\mathrm{cm}$, medida como longitud vertical desde la base del suelo hasta el receptáculo del capítulo; número de hojas $(\mathrm{NH})$ como el total de hojas que presentaban más de $50 \%$ de color verde, indicativo de la presencia de clorofila y de actividad fotosintética; diámetro de tallo (DT) en $\mathrm{cm}$, medida con un vernier a la altura media del tallo; diámetro de capítulo (DC) en $\mathrm{cm}$, como el promedio de dos medidas cruzadas en el capítulo; largo de hoja $(\mathrm{LH})$ en $\mathrm{cm}$, valor promedio de la medición del largo de la nervadura central de tres hojas; ancho de hoja $(\mathrm{AH})$ en $\mathrm{cm}$, valor promedio de la medición de tres hojas de la parte más ancha de la hoja y área foliar (AF) en $\mathrm{cm}^{2}$, determinada con el integrador de área foliar modelo Li-3100.

\section{Obtención de ácido oléico}

La extracción de la grasa de las semillas se realizó de acuerdo a las modificaciones de los métodos de Bligh y Dyer (1959) y de Folch et al. (1957). La muestra pulverizada de semilla de girasol se mezcló con metanol, agua destilada y cloroformo en la proporción 1: 0.9:1.2:0.6. La mezcla se homogenizó por $10 \mathrm{~min}$ a $30 \mathrm{rpm}$ en un agitador rotatorio ATR modelo RKSVD, para después agregar el doble del volumen inicial de cloroformo, agitando la mezcla por 10 min más. Luego se centrifugó por 5 min a 3000 rpm para separ la capa inferior, que está compuesta con cloroformo y la grasa extraida, la cual se filtró con sulfato de sodio activado. La mezcla cloroformo-grasa filtrada se colocó en un rotavapor a $40{ }^{\circ} \mathrm{C}$ para retirar el solvente y obtener la muestra de grasa de las semillas. La metilación de ésteres se realizó con el método 969.33 de la AOAC (1998); para lo cual se mezcló $1 \mathrm{~mL}$ de muestra con $2 \mathrm{ml}$ de $\mathrm{NaOH} 1.0 \mathrm{~N}$ en metanol, para luego agitar la mezcla en un vortex por 1 min y después calentar en baño maría hirviendo por $15 \mathrm{~min}$. La mezcla se dejó enfriar a temperatura de $25{ }^{\circ} \mathrm{C}$ para luego agregar $1 \mathrm{ml}$ de trifluoruro de boro al $14 \%$, agitar en vortex por $1 \mathrm{~min}$ y dejar reposar por $30 \mathrm{~min}$. Posteriormente se le añadió a la mezcla $4 \mathrm{ml}$ de hexano y $1 \mathrm{ml}$ de agua destilada, para después centrifugar a $1000 \mathrm{rpm}$ por $5 \mathrm{~min}$. Luego se separó la capa superior con pipeta Pasteur, y se aforó con hexano a $10 \mathrm{ml}$, almacenando en frasco a $-20{ }^{\circ} \mathrm{C}$ hasta su análisis en un cromatógrafo de gases, para realizar la cuantificación de los ácidos grasos insaturados. Los resultados se obtuvieron en porcentaje y los datos se transformaron por arco seno para realizar el análisis estadístico.

\section{Análisis estadístico}

Se realizó un análisis de varianza combinado para las dos localidades y tres ambientes bajo el modelo $\mathrm{Y}_{i j k}=\mu+\mathrm{G}_{j}+\mathrm{B}(\mathrm{L})_{i(k)}+\mathrm{L}_{k}+(\mathrm{GL})_{j k}$ $+\mathrm{E}_{i j k}$. Donde i es igual a $1,2 \ldots \mathrm{b}$ (bloques dentro de localidades), j es igual a 1, 2 ... g (híbridos), $k$ es igual a $1,2 \ldots$ I (localidades), $\mathrm{Y}_{i j k}$ es la observación fenotípica del j-ésimo híbrido en el i-ésimo bloque de la k-ésima localidad, $\mu$ es la media general, $\mathrm{G}_{j}$ es el efecto del j-ésimo híbrido, $L_{k}$ es el efecto de la k-ésima localidad, $\mathrm{B}(\mathrm{L})_{i(k)}$ es el efecto del i-ésimo bloque dentro de la k-ésima localidad, $(\mathrm{GL})_{j k}$ es el efecto de la interacción del j-ésimo híbrido en la k-ésima localidad y $\mathrm{E}_{i j k}$ es el error experimental. La prueba de Bartlett (Steel y Torrie 1990) se utilizó para determinar homogeneidad de varianzas. Se efectuaron pruebas de comparación de medias por DMS al 0.05 de probabilidad.

\section{Parámetros de estabilidad}

Se realizaron por medio del modelo de Eberhart y Russell (1966) el cual es $\mathrm{Y}_{i j}=\mu_{i}+\beta_{i} \mathbf{I}_{j}+$ $\delta_{i j}+\xi_{i j}$. Donde $\mathrm{Y}_{i j}$ es la media del i-ésimo híbrido en el j-ésimo ambiente $(i=1,2, \ldots, h ; j=1,2, \ldots$ $\mathrm{n}) ; \mu_{i}$ es la media del i-ésimo híbrido sobre todos los ambientes; $\beta_{i}$ el coeficiente de regresión, el cual mide la respuesta de la variable dependiente del i-ésimo híbrido por unidad de cambio de la variable independiente (índice ambiental) en cada diferente ambiente; $\delta_{i j}$ es la desviación de la regresión del 
i-ésimo híbrido en el j-ésimo ambiente; $I_{j}$ es el índice ambiental obtenido como la media de todos los híbridos en el j-ésimo ambiente menos la media general, $\xi_{i j}$ es el efecto del error experimental. Los análisis se realizaron con el paquete SAS V 6.11 (SAS 1998).

\section{RESULTADOS}

El análisis de varianza combinado detectó significancia $(p<0.01)$ en la fuente de variación localidades para todas las variables analizadas, mientras que en la fuente de variación híbridos se encontraron significancias $(p<0.05)$ para las variables largo de hoja y área foliar, y significancia $(p<0.01)$ para rendimiento de grano, ácido oléico, altura de planta, número de hojas y ancho de hoja. También se encontraron diferencias significativas $(p<0.01)$ en la fuente de variación híbrido por localidad para las variables rendimiento de grano y número de hojas, y diferencias significativas $(p<0.05)$ para altura de planta, diámetro de capítulo y largo de hoja (Tabla 1).

En la Tabla 2 se muestran las medias por híbrido, en la que se observan diferencias en el rendimiento de grano, contenido de ácido oléico, altura de planta, número de hojas, área foliar, largo y ancho de hoja. El rendimiento de grano fue de 2.46 a $4.34 \mathrm{t} \mathrm{ha}^{-1}$, presentando el Híbrido 1 el mayor rendimiento, seguido por los híbridos Sabritas 1 e Híbrido 3 con rendimientos de 4.34, 3.67 y 3.41 ton $\mathrm{ha}^{-1}$, respectivamente; los cuales tuvieron rendimientos superiores a la media general de 3.35 ton $\mathrm{ha}^{-1}$. Para el contenido de ácido oléico, las medias fueron de 46.68 a $80.2 \%$, presentando el Híbrido 3, Sabritas 1 e Híbrido 2 valores de 80.2, 79.7 y $75.7 \%$, respectivamente; valores que son superiores a la media general. El híbrido Sabritas 1 sobresalió como el mejor híbrido en cinco de las nueve características evaluadas, mientras que el Híbrido 3 tuvo el mayor contenido de ácido oléico.

El análisis de parámetros de estabilidad (Tabla 3) reveló diferencias significativas $(p<0.05)$ entre los híbridos para el contenido de ácido oléico, altura de planta, número de hojas y área foliar.
Se encontró significancia $(\mathrm{p}<0.05)$ en la interacción de híbridos entre ambiente lineal para altura de planta y número de hojas. En rendimiento de grano, el híbrido Sabritas 1 mostró significancia estadística para la desviación de la regresión. El Híbrido 1 presentó un coeficiente de regresión superior a uno $(\beta \mathrm{i}$ $>1$ ) y el más alto rendimiento. Mientras que Sabritas 1 , tuvo un coeficiente de regresión superior a uno $(\beta \mathrm{i}>1)$, desviación de la regresión significativa, y el segundo mejor rendimiento. Los otros híbridos se clasificaron como estables para rendimiento. Para el contenido de ácido oléico, con excepción de Olisun 1 , todos los híbridos tuvieron coeficientes de regresión superiores a uno $(\beta i>1)$ y desviaciones de regresión no significativas, por lo que se consideraron de buena respuesta en ambientes favorables y consistentes en su contenido de ácido oléico (Tabla 4).

De acuerdo con los índices ambientales de la Tabla 5, las condiciones ambientales que se presentaron en Venecia 2010 fueron favorables para la mayoría de las características evaluadas con excepción del contenido de ácido oléico que tuvo un índice negativo. Mientras que en el ambiente Venecia 2011, los índices ambientales para todas las características fueron positivos. Por el contrario, en el ambiente Durango 2010, los índices ambientales para todas las características fueron negativos. Los más altos contenidos de ácido oléico se obtuvieron en el ambiente Venecia 2011, mientras que el mejor rendimiento de grano fue en el ambiente Venecia 2010.

La estabilidad de los híbridos a través de los ambientes de prueba se presenta en la Tabla 6 , donde se observa que para la mayoría de las características los híbridos fueron estables, con excepción de Sabritas 1 para rendimiento de grano que se clasificó como de buena respuesta en todos los ambientes pero inconsistente, el Híbrido 3 en el número de hojas, el Híbrido 1 en diámetro de tallo y el ancho de hoja, fueron clasificados como de respuesta mejor en ambientes desfavorables y consistentes.

El cálculo de correlaciones fenotípicas entre las variables evaluadas y el contenido de ácido oléico (AO) (Tabla 7) muestran que para el rendimiento 
Tabla 1. Análisis de varianza combinado de seis híbridos de girasol alto oléico evaluados en tres ambientes

\begin{tabular}{llllllllll}
\hline FV & RG & AO & AP & NH & DT & DC & LH & AH & AF \\
\hline LoC & $150.78^{* *}$ & $1347.54^{* *}$ & $13.07^{* *}$ & $1745.32^{* *}$ & $40.50^{* *}$ & $743.32^{* *}$ & $1925.30^{* *}$ & $2234.30^{* *}$ & $31.25^{* *}$ \\
R/L & 1.68 & $410.52^{* *}$ & $0.03^{*}$ & $24.39^{*}$ & 0.58 & 9.20 & 10.76 & 11.52 & $0.42^{*}$ \\
Hib & $4.81^{* *}$ & $2047.17^{* *}$ & $0.13^{* *}$ & $69.93^{* *}$ & 0.22 & 4.00 & $27.30^{*}$ & $24.41^{* *}$ & $0.31^{*}$ \\
HxL & $4.21^{* *}$ & 0.01 & $0.02^{*}$ & $17.55^{* *}$ & 0.35 & $10.89^{*}$ & $12.31^{*}$ & 6.42 & 0.11 \\
Error & 0.79 & 73.02 & 0.01 & 5.01 & 0.25 & 4.30 & 4.75 & 5.06 & 0.11 \\
CV & 26.46 & 12.42 & 6.20 & 7.46 & 20.96 & 11.28 & 9.16 & 9.80 & 21.60
\end{tabular}

$\mathrm{RG}=$ Rendimiento de grano; $\mathrm{AO}=$ ácido oléico; $\mathrm{AP}=$ altura de planta; $\mathrm{NH}=$ número de hojas; $\mathrm{DT}=$ diámetro de tallo; $\mathrm{DC}=$ diámetro de capítulo; $\mathrm{LH}=$ largo de hoja; $\mathrm{AH}=$ ancho de hoja; $\mathrm{AF}=$ área foliar

Tabla 2. Comparación de medias entre los híbridos de girasol alto oléico

\begin{tabular}{llllllllll}
\hline Híbrido & RG & AO & AP & NH & DT & DC & LH & AH & AF \\
\hline Olisun 1 & $2.46 \mathrm{c}$ & $69.65 \mathrm{~b}$ & $1.74 \mathrm{a}$ & $32.70 \mathrm{a}$ & $2.49 \mathrm{a}$ & $18.48 \mathrm{a}$ & $24.63 \mathrm{ab}$ & $23.71 \mathrm{ab}$ & $1.76 \mathrm{a}$ \\
Olisun 2 & $3.24 \mathrm{~b}$ & $46.68 \mathrm{~d}$ & $1.54 \mathrm{~d}$ & $29.11 \mathrm{~b}$ & $2.48 \mathrm{a}$ & $17.62 \mathrm{a}$ & $24.28 \mathrm{ab}$ & $23.09 \mathrm{~b}$ & $1.62 \mathrm{ab}$ \\
Sabritas 1 & $3.67 \mathrm{ab}$ & $79.70 \mathrm{a}$ & $1.64 \mathrm{bc}$ & $26.55 \mathrm{c}$ & $2.23 \mathrm{a}$ & $17.93 \mathrm{a}$ & $25.90 \mathrm{a}$ & $25.15 \mathrm{a}$ & $1.57 \mathrm{abc}$ \\
Híbrido 1 & $4.34 \mathrm{a}$ & $60.62 \mathrm{c}$ & $1.70 \mathrm{ab}$ & $32.84 \mathrm{a}$ & $2.58 \mathrm{a}$ & $19.15 \mathrm{a}$ & $23.55 \mathrm{~b}$ & $22.05 \mathrm{bc}$ & $1.64 \mathrm{ab}$ \\
Híbrido 2 & $3.01 \mathrm{bc}$ & $75.70 \mathrm{ab}$ & $1.61 \mathrm{~cd}$ & $29.04 \mathrm{~b}$ & $2.27 \mathrm{a}$ & $18.23 \mathrm{a}$ & $22.87 \mathrm{bc}$ & $22.80 \mathrm{bc}$ & $1.39 \mathrm{bc}$ \\
Híbrido 3 & $3.41 \mathrm{~b}$ & $80.20 \mathrm{a}$ & $1.45 \mathrm{e}$ & $29.55 \mathrm{~b}$ & $2.47 \mathrm{a}$ & $18.90 \mathrm{a}$ & $21.54 \mathrm{c}$ & $20.98 \mathrm{c}$ & $1.32 \mathrm{c}$ \\
DMS & 0.73 & 7.02 & 0.08 & 1.84 & 0.41 & 1.70 & 1.79 & 1.85 & 0.27 \\
$\mu$ & 3.35 & 68.75 & 1.61 & 29.96 & 2.42 & 18.38 & 23.79 & 22.96 & 1.55 \\
\hline
\end{tabular}

RG = Rendimiento de grano; $\mathrm{AO}=$ ácido oléico; $\mathrm{AP}=$ altura de planta; $\mathrm{NH}=$ número de hojas; $\mathrm{DT}=$ diámetro de tallo; $\mathrm{DC}=$ diámetro de capítulo; $\mathrm{LH}=$ largo de hoja; $\mathrm{AH}=$ ancho de hoja; $\mathrm{AF}=$ área foliar

Tabla 3. Análisis de varianza de los parámetros de estabilidad.

\begin{tabular}{llllllllll}
\hline F.V. & RG & AO & AP & NH & DT & DC & LH & AH & AF \\
\hline Total & 5.41 & 1.85 & 0.39 & 59.02 & 1.27 & 23.75 & 60.42 & 68.42 & 0.96 \\
Híbridos (H) & 1.22 & $502.77^{*}$ & $0.03^{*}$ & $17.49^{*}$ & 0.07 & 1.00 & 6.83 & 6.11 & $0.08 *$ \\
Ambientes (A) & 7.16 & 52.99 & 0.54 & 76.3 & 1.77 & 33.23 & 82.75 & 94.39 & 1.32 \\
A (lineal) & 75.26 & 633.92 & 6.54 & 872.2 & 20.21 & 371.63 & 962.26 & 1116.66 & 15.64 \\
H*A (lineal) & 0.49 & 0.27 & $0.01^{*}$ & $5.98 *$ & 0.02 & 2.24 & 2.31 & 1.91 & 0.03 \\
Desviación Conjunta & 1.37 & 0.12 & 0.00 & 2.33 & 0.17 & 2.66 & 3.20 & 1.09 & 0.02 \\
Olisun 1 & 2.29 & 0.03 & 0.01 & 2.11 & 0.04 & 1.72 & 3.69 & 0.12 & 0.00 \\
Olisun 2 & 0.18 & 0.04 & 0.00 & 1.34 & 0.28 & 8.32 & 1.01 & 1.81 & 0.01 \\
Sabritas 1 & $3.21^{*}$ & 0.03 & 0.00 & 0.87 & 0.26 & 2.31 & 2.49 & 0.22 & 0.02 \\
Híbrido 1 & 1.80 & 0.50 & 0.00 & 9.52 & 0.00 & 2.04 & 3.45 & 0.00 & 0.10 \\
Híbrido 2 & 0.53 & 0.08 & 0.00 & 0.16 & 0.03 & 1.32 & 8.49 & 4.07 & 0.01 \\
Híbrido 3 & 0.22 & 0.05 & 0.00 & 0.00 & 0.43 & 0.27 & 0.09 & 0.30 & 0.00 \\
Error Conjunto & 0.79 & 73.00 & 0.01 & 5.01 & 0.25 & 4.30 & 4.75 & 5.06 & 0.11 \\
\hline
\end{tabular}

$\mathrm{RG}=$ Rendimiento de grano; $\mathrm{AO}=$ ácido oléico; $\mathrm{AP}=$ altura de planta; $\mathrm{NH}=$ número de hojas; $\mathrm{DT}=$ diámetro de tallo; $\mathrm{DC}=$ diámetro de capítulo; $\mathrm{LH}=$ largo de hoja; $\mathrm{AH}=$ ancho de hoja; $\mathrm{AF}=$ área foliar.

de grano (RG) se correlacionó de forma positiva y significativa $(p<0.01)$ con el largo $(L=0.50)$ y ancho $(A=0.49)$ de hoja. Se observaron correlaciones significativas $(p<0.05)$ entre el diámetro de tallo y número de hojas (0.48), largo de hoja y diámetro de capítulo (0.47), y diámetro de capítulo y altura de planta $(-0.41)$. Correlaciones altamente significativas $(\mathrm{p}<0.01)$ se observaron entre número de hojas y área foliar (0.53), diámetro de capítulo y diámetro de tallo (0.62), diámetro de capítulo y ancho de hoja (0.59) y área foliar (0.62), entre largo y ancho de hoja (0.88) y área foliar (0.75), y ancho de hoja con el área foliar (0.85).

\section{DISCUSIÓN}

El efecto ambiental es un factor que influye en la respuesta fenotípica de los cultivos, el rendimiento de grano y contenido de aceite (Tabrizi 2012). En el análisis de varianza combinado (Tabla 1) se observó efecto significativo del ambiente de cada localidad sobre el comportamiento de los híbridos evaluados. La significancia observada para los híbridos, señala variabilidad genética entre ellos, la significancia en la interacción híbrido $x$ ambiente para rendimiento, altura de planta, número de hojas, 
Tabla 4. Parámetros de estabilidad de los híbridos evaluados.

\begin{tabular}{llrrrrrr}
\hline \multirow{2}{*}{ Variable } & Parámetro & \multicolumn{7}{c}{ Híbrido } \\
\cline { 2 - 7 } & & Olisun 1 & Olisun 2 & Sabritas 1 & Híbrido 1 & Híbrido 2 & Híbrido 3 \\
\hline $\mathrm{RG}$ & $\beta \mathrm{i}$ & 0.65 & 1.02 & 1.04 & 1.24 & 0.93 & 1.11 \\
& $\mathrm{~S}^{2} \mathrm{di}$ & 1.50 & -0.61 & $2.42^{*}$ & 1.01 & -0.26 & -0.57 \\
$\mathrm{AO}$ & $\beta \mathrm{i}$ & 0.98 & 1.03 & 1.01 & 1.02 & 1.05 & 1.04 \\
& $\mathrm{~S}^{2} \mathrm{di}$ & -72.97 & -72.96 & -72.97 & -72.50 & -72.92 & -72.95 \\
$\mathrm{AP}$ & $\beta \mathrm{i}$ & 1.09 & 1.07 & 0.98 & 1.04 & 0.97 & 0.86 \\
& $\mathrm{~S}^{2} \mathrm{di}$ & 0.00 & -0.01 & -0.01 & -0.01 & -0.01 & -0.01 \\
$\mathrm{DT}$ & $\beta \mathrm{i}$ & 1.11 & 1.15 & 1.09 & 1.14 & 0.85 & $0.66 *$ \\
& $\mathrm{~S}^{2} \mathrm{di}$ & -2.90 & -3.67 & -4.14 & 4.51 & -4.85 & -5.01 \\
$\mathrm{DC}$ & $\beta \mathrm{i}$ & 0.74 & 1.10 & 1.30 & 1.01 & 0.88 & 0.97 \\
& $\mathrm{~S}^{2} \mathrm{di}$ & -2.58 & 4.02 & -1.99 & -2.26 & -2.98 & -4.03 \\
$\mathrm{LH}$ & $\beta \mathrm{i}$ & 0.96 & 1.13 & 0.99 & 1.05 & 0.79 & 1.08 \\
& $\mathrm{~S}^{2} \mathrm{di}$ & -1.06 & -3.74 & -2.26 & -1.30 & 3.74 & -4.66 \\
$\mathrm{AH}$ & $\beta \mathrm{i}$ & 0.82 & 1.04 & 1.11 & $0.98 *$ & 1.00 & 1.05 \\
& $\mathrm{~S}^{2} \mathrm{di}$ & -4.94 & -3.25 & -4.84 & -5.06 & -0.99 & -4.76 \\
$\mathrm{AF}$ & $\beta \mathrm{i}$ & 1.08 & 1.08 & 0.90 & 1.12 & 0.86 & 0.96 \\
& $\mathrm{~S}^{2} \mathrm{di}$ & -0.11 & -0.10 & -0.09 & -0.01 & -0.10 & -0.11 \\
\hline
\end{tabular}

$\mathrm{RG}=$ Rendimiento de grano; $\mathrm{AO}=$ ácido oléico; $\mathrm{AP}=$ altura de planta; $\mathrm{NH}=$ número de hojas; $\mathrm{DT}=$ diámetro de tallo; $\mathrm{DC}=$ diámetro de capítulo; $\mathrm{LH}=$ largo de hoja; $\mathrm{AH}=$ ancho de hoja; $\mathrm{AF}$ = área foliar.

Tabla 5. Índices ambientales de las características evaluadas.

\begin{tabular}{lrrrrrrrrr}
\hline Ambiente & RG & AO & AP & NH & DT & DC & LH & AH & AF \\
\hline Venecia 2010 & 1.8 & -6.0 & 0.51 & 4.39 & 0.04 & 3.55 & 2.64 & 5.04 & 0.43 \\
Durango 2010 & -2.8 & -2.0 & -0.84 & -9.82 & -1.31 & -6.41 & -9.98 & -11.12 & -1.29 \\
Venecia 2011 & 1.0 & 8.0 & 0.33 & 5.43 & 1.28 & 2.85 & 7.33 & 6.07 & 0.85 \\
\hline RG = Rendimiento de grano; AO = ácido oléico; AP = altura de planta; & NH = número \\
de hojas; DT = diámetro de tallo; DC = diámetro de capítulo; LH = largo de hoja; AH = \\
ancho de hoja; AF = área foliar.
\end{tabular}

Tabla 6. Descripción de la estabilidad de los híbridos de girasol evaluados.

\begin{tabular}{|c|c|c|c|c|c|c|c|c|c|}
\hline Híbrido & RG & $\mathrm{AO}$ & AP & $\mathrm{NH}$ & DT & DC & $\mathrm{LH}$ & $\mathrm{AH}$ & AF \\
\hline Olisun 1 & Estable & Estable & Estable & Estable & Estable & Estable & Estable & Estable & Estable \\
\hline Olisun 2 & Estable & Estable & Estable & Estable & Estable & Estable & Estable & Estable & Estable \\
\hline Sabritas 1 & BRAI & Estable & Estable & Estable & Estable & Estable & Estable & Estable & Estable \\
\hline Híbrido 1 & Estable & Estable & Estable & Estable & RMADC & Estable & Estable & RMADC & Estable \\
\hline Híbrido 2 & Estable & Estable & Estable & Estable & Estable & Estable & Estable & Estable & Estable \\
\hline Híbrido 3 & Estable & Estable & Estable & RMADC & Estable & Estable & Estable & Estable & Estable \\
\hline
\end{tabular}

diámetro de capítulo y largo de hoja, señala una respuesta diferente de los híbridos a las variaciones ambientales en las localidades (Hassan et al. 2013). Lo anterior, plantea la necesidad de investigar la respuesta diferencial de los híbridos a los ambientes (Van der Merwe et al. 2013, Bhoite et al. 2010), ya que de acuerdo a Geisel (2011), los organismos responden a los factores ambientales mediante, cambios metabólicos y morfológicos. Una alternativa para minimizar la interacción genotipo $x$ ambiente es seleccionar genotipos estables para am- bientes de interés a través del análisis de estabilidad, que relaciona los efectos de genotipos en forma independiente dentro de cada ambiente, con los efectos ambientales (Tabrizi 2012, Orona et al. 2013).

En la comparación de medias (Tabla 2), se observa alta variabilidad entre los híbridos para la mayoría de las variables evaluadas, con excepción del diámetro de tallo y capítulo. Los girasoles con alto contenido de ácido oléico son aquéllos en los que sus contenidos se encuentran entre 75 y $90.7 \%$ 
Tabla 7. Correlaciones fenotípicas entre nueve caracteres evaluados en híbridos de girasol alto oléico.

\begin{tabular}{lrrrrrrrrr}
\hline & AO & RG & AP & NH & DT & DC & L & A & AF \\
\hline AO & 1.00 & & & & & & & & \\
RTO & -0.28 & 1.00 & & & & & & & \\
AP & -0.26 & 0.10 & 1.00 & & & & & & \\
NH & -0.08 & -0.28 & 0.29 & 1.00 & & & & & \\
DT & 0.10 & -0.31 & -0.19 & $0.48^{*}$ & 1.00 & & & & \\
DC & -0.01 & 0.07 & $-0.41^{*}$ & 0.29 & $0.62^{* *}$ & 1.00 & & & \\
L & 0.09 & $0.50^{* *}$ & -0.01 & -0.11 & 0.01 & $0.47^{*}$ & 1.00 & & \\
A & -0.02 & $0.49 * *$ & 0.03 & 0.06 & 0.16 & $0.59^{* *}$ & $0.88^{* *}$ & 1.00 & \\
AF & -0.04 & 0.29 & 0.18 & $0.53^{* *}$ & 0.38 & $0.62^{* *}$ & $0.75^{* *}$ & $0.85^{* *}$ & 1.00 \\
\hline
\end{tabular}

*: Significativo $(\mathrm{P}<0.05)$; **: Altamente significativo $(\mathrm{P}<0.01), \mathrm{RG}=$ Rendimiento de grano; $\mathrm{AO}=$ ácido oléico; $\mathrm{AP}=$ altura de planta; $\mathrm{NH}=$ número de hojas; $\mathrm{DT}=$ diámetro de tallo; $\mathrm{DC}=$ diámetro de capítulo; $\mathrm{LH}=$ largo de hoja; $\mathrm{AH}=$ ancho de hoja; $\mathrm{AF}=$ área foliar.

(Ferfuia et al. 2015). Por lo que tres de los híbridos evaluados se pueden considerar como de alto oléico, ya que sus contenidos oscilaron entre 75.70 y 80.20 $\%$, valores de 66.97 a $73.04 \%$ reportados por Van der Merwe et al. (2013). Mientras que Agüero et al. (1999) identificaron híbridos comerciales de girasol alto oléico que tenían contenidos de 79.9. Para rendimiento de grano la media fue de $3.35 \mathrm{t} \mathrm{ha}^{-1}$, valores que son superiores a la media nacional de $1.12 \mathrm{t} \mathrm{ha}^{-1}$ y la media del estado de Durango de 1.85 ton ha ${ }^{-1}$ (SIAP 2014), pero no superan las 4.5 t ha ${ }^{-1}$ reportadas por Ávila et al. (2007) para híbridos. Por lo anterior, y considerando que cuatro de los híbridos son experimentales se puede considerar que el rendimiento promedio de los híbridos evaluados y contenido de ácido oléico es aceptable.

La significancia encontrada en la interacción híbrido $x$ ambiente para algunas variables, justificó el análisis de varianza de estabilidad (Tabla 3). Para los híbridos se observó variabilidad genética entre ellos, lo que permitió detectar aquellos con potencial genético, mientras que la significancia en la interacción híbrido $x$ ambiente lineal indica diferencias en los coeficientes de regresión (Akcura et al. 2005), lo que señala que los híbridos responden de forma diferencial en ambientes malos y buenos. La significancia observada en el híbrido Sabritas 1 para rendimiento, señala que su respuesta es inconsistente con los cambios ambientales. Para realizar selección se debe considerar el rendimiento y la estabilidad de su comportamiento (Da Silva, 2014). Todos los híbridos tienen el potencial de expresar altos rendimientos de grano y de ácido oléico. Pero el contenido de ácido oléico está en función de la enzima oleil-CoA-desaturasa que depende de la temperatura del medio ambiente (Agüero et al. 1999). Al respecto Rolletschek et al. (2007), encontraron que al aumentar la temperatura ambiental de 10 a $40{ }^{\circ} \mathrm{C}$, se limita la actividad de la enzima, lo que evita la desaturación del ácido oléico a linoléico, pudiendo esta situación explicar el efecto benéfico de las temperaturas de Venecia.

De acuerdo con Eberhart y Rusell (1966) los híbridos estables, son aquellos con $\beta \mathrm{i}=1$ y $\mathrm{Sdi}^{2}=0$, que responden de acuerdo a las fluctuaciones ambientales y son predecibles en su rendimiento. Al respecto Akcura et al. (2006) señalan que valores de regresión superiores a 1.0 describen genotipos con mayor sensibilidad a los cambios ambientales, con mayor capacidad de adaptación a los ambientes de alto rendimiento. Mientras que coeficientes de regresión menores de 1.0 tienen mayor resistencia a los cambios ambientales. En la Tabla 4 se puede apreciar que para rendimiento de grano, con excepción de Sabritas 1, todos los híbridos se clasifican como estables $\left(b=1\right.$ y $\left.\mathrm{Sdi}^{2}=0\right)$, lo que indica que tienen buena respuesta en todos los ambientes y son consistentes. El híbrido Sabritas 1 en la variable rendimiento de grano se clasificó como inconsistente $\left(\mathrm{Sdi}^{2}>0\right)$ ya que su rendimiento se vuelve impredecible si el ambiente no presenta las condiciones adecuadas. Para contenido de ácido oléico y con excepción de Olisun 1 , los demás híbridos se consideraron estables y consistentes $(b>1.0$ y $\left.\mathrm{Sdi}^{2}=0\right)$.

De acuerdo con los índices ambientales (Tabla 
5), el mejor ambiente fue Venecia 2011 para todas las variables. Para rendimiento de grano, los dos ambientes en Venecia presentaron las mejores condiciones para el cultivo de girasol. Las condiciones ambientales presentes en el ambiente de Durango no fueron favorables para el contenido de ácido oléico, debido a que las temperaturas son frescas, lo que provoca disminución en el llenado de grano, lo que impacta en el rendimiento y producción de ácido oléico (Xiang-Min et al. 2014). En la localidad de Venecia 2011 se obtuvo un índice mayor para el ácido oléico, y la fecha de siembra fue más temprana que en los otros ciclos, coincidiendo el llenado de grano con temperaturas altas, mientras que en el ciclo 2010 en Venecia, la temperatura máxima que se presentó en los meses críticos de floración, llenado de grano y madurez fisiológica fue de $36.9{ }^{\circ} \mathrm{C}$, con promedio de $33^{\circ} \mathrm{C}$, y en 2011 la temperatura máxima fue de $38.8^{\circ} \mathrm{C}$, con promedio de $34.7{ }^{\circ} \mathrm{C}$, favoreciendo éstas últimas la producción de ácido oléico. De acuerdo con Abd El-Mohsen (2012) los rendimientos de grano de girasol y aceite se reducen cuando las fechas normales de siembra se retrasan debido a que se presentan temperaturas por debajo del óptimo en la etapa reproductiva del cultivo. La clasificación de la estabilidad de los híbridos (Tabla 6) considerando el valor promedio de las características evaluadas y los parámetros de estabilidad, señalan que los híbridos en general se consideraron estables y predecibles en su respuesta a los cambios ambientales. Sabritas 1 se clasificó como de buena respuesta en todos los ambientes pero inconsistente para rendimiento de grano, el Híbrido
1 fue el mejor para rendimiento de grano y estable, mientras que el Híbrido 3 fue el mejor para contenido de ácido oléico y estable. Los cuales se pueden considerar competitivos, ya que su rendimiento de grano superan a la media nacional y del estado de Durango (SIAP 2014).

La correlación significativa entre el rendimiento de grano con largo y ancho de hoja, con área foliar indica que es posible obtener cultivares más productivos (Yasin y Singh 2010). Estas correlaciones entre el largo y ancho de hoja, y el área foliar podrían aumentar la producción de biomasa al aumentar la fijación de carbono (Tyagi y Khan 2013). La importancia del área foliar ha sido demostrada en girasol y otros cultivos, ya que el mantenimiento por más tiempo del área foliar fotosintética durante la etapa reproductiva tiene un impacto en el rendimiento (Moschen et al. 2016).

\section{CONCLUSIONES}

Al considerar el rendimiento de grano, contenido de ácido oléico y estabilidad de los híbridos, sobresalen el Híbrido 3 y Sabritas 1, los cuales tienen el potencial comercial. La localidad de Venecia es mejor para la siembra de girasol, principalmente por las condiciones agroclimáticas que propician altos contenidos de ácido oléico. Para tener una alta producción de ácido oléico en el cultivo de girasol, además de sembrar materiales genéticos mejorados y adaptados, se requiere poner énfasis en la fecha de siembra, para garantizar temperaturas adecuadas para la formación y llenado de grano.

\section{LITERATURA CITADA}

Abd El-Mohsen AA (2012) Analyzing and modeling the relationship between yield and yield components in sunflower under different planting dates. Journal of Plant Breeding and Crop Science 4: 125-135.

Akcura M, Kaya Y, Taner S, Ayranci R (2006) Parametric stability analyses for grain yield of durum wheat. Plant Soil Environment 52: 254-261.

Akcura M, Kaya Y, Taner S (2005) Genotype-environment interaction and phenotypic stability analysis for grain yield of durum wheat in the central anatolian Region. Turkish Journal Of Agriculture and Forestry 29: 369-375.

Alberio C, Izquierdo NG, Galella T, Zuil S, Reid R, Zambelli A, et al. (2016) A new sunflower high oleic mutation confers stable oil grain fatty acid composition across environments. European Journal of 
Agronomy 73: 25-33.

Ali MA, Najmaldien AHA, Latip RA, Othman NH, Majid FAA, Salleh LM (2013) Effect of heating at frying temperature on the quality characteristics of regular and high-oleic acid sunflower oils. Acta scientiarum Polonorum : Technologia Alimentaria 12: 159-167.

Agüero ME, Pereyra VR, Aguirrezábal LAN, Lúquez J (1999) Rendimiento de grano y porcentaje de aceite de híbridos de girasol "alto oléico" cultivados en Argentina. Agriscientia 16: 49-53.

AOAC. 1998. Fatty acids in oils and fats, Method 969.33 Preparation of methyl esters, boron trifluoride method. Vol II. edited by P. Cunniff. Gaitherburg, Maryland, USA, Chapter 41. 17p.

Ávila, J, Díaz A, De La Cruz R, Moreno N, Romero D, Cáceres R, Gutiérrez L, Flores R (2007) Evaluación comparativa de híbridos de girasol (Helianthus annus L.) en dos zonas productoras de Venezuela. Bioagro 19: 3-9.

Balalić I, Zorić M, Miklić V, Dušanić N, Terzić S, Radić V (2011) Non-parametric stability analysis of sunflower oil yield trials. HELIA 34: 67-78.

Bhoite KD, Kanwade DG, Gadekar DA (2010) Stability parameters in seed yield in sunflower hybrids. Agricultural Science Digest 30: 152-153.

Bligh EG, Dyer WJ (1959) A rapid method of total lipid extraction and purification. Canadian Journal of Biochemistry and Physiology 37: 911-917.

Da Silva PR, Bisognin DA, Locatelli AB, Storck L (2014) Adaptability and stability of corn hybrids grown for high grain yield. Acta Scientiarum Agronomy 36: 175-181.

Eberhart SA, Russell WA (1966) Stability parameters for comparing varieties. Crop Science 6: 36-40.

Ferfuia C, Turi M, Vannozzi GP (2015) Variability of seed fatty acid composition to growing degree-days in high oleic acid sunflower genotypes. HELIA 38: 61-78.

Folch JM, Lessy M, Sloan SH (1957) A simple method for isolation and purification of total lipids from animal tissues. Journal of Biological Chemistry 226: 497-509.

Geisel N (2011) Constitutive versus responsive gene expression strategies for growth in changing environments. PLOS ONE 6: e27033

Hassan MS, Mohamed GIA, El-Said RAR (2013) Stability analysis for grain yield and its components of some durum wheat genotypes (Triticum durum L.) under different environments. Asian Journal of Crop Science 5: 179-189.

INEGI (2008) Atlas Nacional Interactivo de México. Estado de Durango. Centro distribuidor de metadatos http://www.inegi.org.mx. Fecha de consulta 10 de marzo de 2016.

INIFAP (2015) Paquetes tecnológicos. Girasol. INIFAP-Chihuahua. http://sites.securemgr.com/folder11341/ index.cfm?id=921810\&fuseaction =browse\&pageid=51. Fecha de consulta 25 de noviembre de 2015.

Moschen S, Bengoa LS, Di Rienzo JA, Caro MP, Tohge T, Watanabe M, et al. (2016) Integrating transcriptomic and metabolomic analysis to understand natural leaf senescence in sunflower. Plant Biotechnology Journal 14: 719-734.

Orona CF, Medina MJ, Tucuch FM, Soto RJM, Almeyda LIH (2013) Parámetros de estabilidad en rendimiento y adaptabilidad de 25 genotipos de arroz en Campeche, México. Phyton Revista Internacional de Botánica Experimental 82: 255-261 
Rolletschek H, Borisjuk L, Sánchez-García A, Gotor C, Romero LC, Martínez-Rivas JM, et al. (2007) Temperature-dependent endogenous oxygen concentration regulates microsomal oleate desaturase in developing sunflower seeds. Journal of Experimental Botany 58: 3171-3181.

SAS (1998) Statistical Analysis System. User's guide. Version 6.11. SAS Institute Inc. Cary, NC, USA.

SIAP (2014) Cierre de la producción agrícola por cultivo. Servicio de Información Agroalimentaria y Pesquera http://www.siap.gob.mx/cierre-de-la-produccion-agricola-por-cultivo/. Fecha de consulta 10 de marzo de 2016.

Sobrino E, Tarquis AM, Cruz Díaz M (2003) Modeling the oleic acid content in sunflower oil. Agronomy Journal 95: 329-334.

Steel RGD, Torrie JH (1990) Bioestadística: Principios y procedimientos. $2^{\text {a }}$ Ed. McGraw Hill, Interamericana de México. México. 622p.

Tabrizi HZ (2012) Genotype by environment interaction and oil yield stability analysis of six sunflower cultivars in Khoy, Iran. Advances in Environmental Biology 6: 227-231.

Turhan H, Citak N, Pehlivanoglu H, Mengul Z (2010) Effects of ecological and topographic conditions on oil content and fatty acid composition in sunflower. Bulgarian Journal of Agricultural Science 16: 553-558.

Tyagi SD, Khan MH (2013) Correlation and path coefficient analysis for seed yield in sunflower (Helianthus annuus L.). International Journal of Agricultural Research, Sustainability, and Food Sufficiency 1: 7-13.

Van der Merwe R, Labuschagne MT, Herselman L, Hugo A (2013) Stability of seed oil quality traits in high and mid-oleic acid sunflower hybrids. Euphytica 193: 157-168.

Xiang-Min P, Seong YC, Young SJ, Yoon-Sup S, Jong-Wook C, Sok-Young L, et al. (2014) Effect of genotype, growing year and planting date on agronomic traits and chemical composition in sunflower (Helianthus annuus L.) Germplasm. Plant Breeding and Biotechnology 2: 35-47.

Yasin AB, Singh S (2010) Correlation and path coefficient analyses in sunflower. Journal of Plant Breeding and Crop Science 2: 129-133. 